\title{
Strategies for cellular deconvolution in human brain RNA
}

\section{sequencing data [version 1; peer review: 1 approved, 1}

\section{approved with reservations]}

\author{
Olukayode A. Sosina (D1), Matthew N. Tran2,3, Kristen R. Maynard2, Ran Tao², \\ Margaret A. Taub1, Keri Martinowich2,4,5, Stephen A. Semick (iD2, Bryan C. Quach6, \\ Daniel R. Weinberger2-4,7, Thomas Hyde 2,4,7, Dana B. Hancock6 ${ }^{6}$ Joel E. Kleinman2,4, \\ Jeffrey T. Leek ${ }^{1}$, Andrew E. Jaffe (iD)1-5,8
}

\footnotetext{
${ }^{1}$ Department of Biostatistics, Bloomberg School of Public Health, Johns Hopkins University, Baltimore, USA

${ }^{2}$ Lieber Institute for Brain Development, Baltimore, USA

${ }^{3}$ Department of Genetic Medicine, Johns Hopkins Medicine, Baltimore, USA

${ }^{4}$ Department of Psychiatry and Behavioral Sciences, Johns Hopkins Medicine, Baltimore, USA

${ }^{5}$ Department of Neuroscience, School of Medicine, Johns Hopkins Medicine, Baltimore, USA

${ }^{6}$ Center for Omics Discovery and Epidemiology, RTI International, Durham, USA

${ }^{7}$ Department of Neurology, Johns Hopkins Medicine, Baltimore, USA

${ }^{8}$ Department of Mental Health, Bloomberg School of Public Health, Johns Hopkins University, Baltimore, USA
}

V1 First published: 04 Aug 2021, 10:750

https://doi.org/10.12688/f1000research.50858.1

Latest published: 04 Aug 2021, 10:750

https://doi.org/10.12688/f1000research.50858.1

\section{Abstract}

Background: Statistical deconvolution strategies have emerged over the past decade to estimate the proportion of various cell populations in homogenate tissue sources like brain using gene expression data. However, no study has been undertaken to assess the extent to which expression-based and DNAm-based cell type composition estimates agree.

Results: Using estimated neuronal fractions from DNAm data, from the same brain region (i.e., matched) as our bulk RNA-Seq dataset, as proxies for the true unobserved cell-type fractions (i.e., as the gold standard), we assessed the accuracy (RMSE) and concordance $\left(R^{2}\right)$ of four reference-based deconvolution algorithms: Houseman, CIBERSORT, non-negative least squares (NNLS)/MIND, and MuSiC. We did this for two cell-type populations - neurons and non-neurons/glia using matched single nuclei RNA-Seq and mismatched single cell RNASeq reference datasets. With the mismatched single cell RNA-Seq reference dataset, Houseman, MuSiC, and NNLS produced concordant (high correlation; Houseman $\mathrm{R}^{2}=0.51,95 \% \mathrm{CI}[0.39,0.65]$; MuSiC $\mathrm{R}^{2}=$ $0.56,95 \%$ CI $[0.43,0.69]$; NNLS R ${ }^{2}=0.54,95 \%$ CI $[0.32,0.68]$ ) but biased (high RMSE, $\geq 0.35$ ) neuronal fraction estimates. CIBERSORT produced more discordant (moderate correlation; $\mathrm{R}^{2}=0.25,95 \% \mathrm{CI}$ $[0.15,0.38]$ ) neuronal fraction estimates, but with less bias (low RSME,

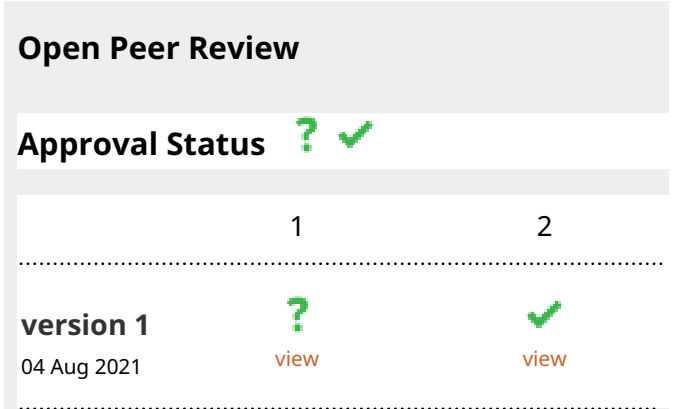

1. Kathryn Roeder, Carnegie Mellon University, Pittsburgh, USA

Jiebiao Wang (D), University of Pittsburgh,

Pittsburgh, USA

2. Arjun Bhattacharya (D), University of

California, Los Angeles, Los Angeles, USA

Any reports and responses or comments on the article can be found at the end of the article. 
0.09). Using the matched single nuclei RNA-Seq reference dataset did not eliminate bias (MUSiC RMSE $=0.17$ ).

Conclusions: Our results together suggest that many existing RNA deconvolution algorithms estimate the RNA composition of homogenate tissue, e.g. the amount of RNA attributable to each cell type, and not the cellular composition, which relates to the underlying fraction of cells.

Keywords

Statistical deconvolution, DNA methylation, RNA-seq, single nucleus

RNA-seq

\section{“incf}

This article is included in the INCF gateway.

Corresponding author: Andrew E. Jaffe (andrew.jaffe@libd.org)

Author roles: Sosina OA: Data Curation, Formal Analysis, Investigation, Methodology, Software, Visualization, Writing - Original Draft Preparation, Writing - Review \& Editing; Tran MN: Data Curation, Formal Analysis, Methodology, Resources, Writing - Original Draft Preparation, Writing - Review \& Editing; Maynard KR: Data Curation, Formal Analysis, Investigation, Methodology, Resources, Validation, Writing - Review \& Editing; Tao R: Data Curation, Investigation, Resources; Taub MA: Formal Analysis, Investigation, Methodology; Martinowich K: Investigation, Resources, Writing - Review \& Editing; Semick SA: Formal Analysis, Investigation, Resources, Writing - Review \& Editing; Quach BC: Formal Analysis, Investigation, Resources, Writing - Review \& Editing; Weinberger DR: Resources, Writing - Review \& Editing; Hyde T: Resources; Hancock DB: Funding Acquisition, Project Administration, Resources, Supervision, Writing - Review \& Editing; Kleinman JE: Resources; Leek JT: Conceptualization, Formal Analysis, Investigation, Methodology, Supervision, Writing - Original Draft Preparation, Writing - Review \& Editing; Jaffe AE: Conceptualization, Formal Analysis, Funding Acquisition, Investigation, Methodology, Project Administration, Supervision, Writing - Original Draft Preparation, Writing Review \& Editing

Competing interests: No competing interests were disclosed.

Grant information: K.M., R.T., S.A.S., D.B.H., and A.E.J. were partially supported by the National Institute on Drug Abuse (R01DA042090); K.R.M., K.M. and A.E.J. were partially supported by the National Institute of Mental Health (R01MH123183).

The funders had no role in study design, data collection and analysis, decision to publish, or preparation of the manuscript.

Copyright: $\odot 2021$ Sosina OA et al. This is an open access article distributed under the terms of the Creative Commons Attribution License, which permits unrestricted use, distribution, and reproduction in any medium, provided the original work is properly cited.

How to cite this article: Sosina OA, Tran MN, Maynard KR et al. Strategies for cellular deconvolution in human brain RNA sequencing data [version 1; peer review: 1 approved, 1 approved with reservations] F1000Research 2021, 10:750

https://doi.org/10.12688/f1000research.50858.1

First published: 04 Aug 2021, 10:750 https://doi.org/10.12688/f1000research.50858.1 


\section{Introduction}

Homogenate tissues like brain and blood contain a mixture of cell types which can each have unique genomic profiles, and these mixtures of cell types, termed "cellular composition", can vary across samples (Jaffe and Irizarry 2014). The importance of considering cellular composition within heterogeneous tissue sources has been highlighted in epigenetics research over the past several years (Houseman et al. 2012; Montaño et al. 2013; Jaffe and Irizarry 2014), as, generally, failure to account for cellular composition when analyzing heterogeneous tissue sources can increase both false positives and negatives (Michels et al. 2013). Previous work has identified widespread epigenetic differences between neurons and glia using DNA methylation (DNAm) data (Montaño et al. 2013; Guintivano et al. 2013), and false positives may arise when there are cellular composition differences associated with dissection variability, disease, normal development or any other outcome of interest. For example, loss of neurons (or glia) because of disease may cause spurious loci associations with illness that stem solely from differing cellular compositions between disease states, or cell-type specific biological differences may exist that become more difficult to detect in the presence of unaffected cell types.

Statistical algorithms estimate the relative or absolute amounts of each cell type in the homogenate tissue data. These so called "cellular deconvolution" algorithms have been especially popular using DNAm data (Houseman et al. 2014) as DNAm levels are constrained between 0 and 1 and are binary within single cells (i.e. individual CpGs are either methylated or unmethylated). These deconvolution algorithms can be classified into two general types, termed "referenced-based" and "reference-free" (Houseman et al. 2014; Avila Cobos et al. 2018). Reference-free approaches only require as input an estimate of the number of potential cell types in a particular dataset (which can be non-trivial), and return latent components that preferentially capture cellular heterogeneity that can be adjusted for in differential methylation analysis (Houseman et al. 2014; Jaffe and Irizarry 2014; Rahmani et al. 2016). However, these approaches do not return fractions of cells and may capture potential batch effects in addition to cellular composition. Conversely, reference-based approaches require cell type-specific genomic profiles for each cell type of interest as an input and return the relative fraction of each input cell type for each queried bulk sample (Houseman et al. 2012), akin to an in silico cell counter. This class of algorithms therefore requires the generation of potentially many pure cell populations, which are typically generated from flow cytometry for applications to DNAm data from bulk tissue.

While DNAm data can generate accurate absolute cell fractions in homogenate brain tissue (Amanda J. Price et al. 2019; Guintivano et al. 2013; Montaño et al. 2013), there are several important considerations limiting more widespread application. First, RNA and gene expression profiling has been much more popular in postmortem brain studies, with more samples profiled with RNA sequencing (RNA-seq) than DNAm microarrays or sequencing. Secondly, the two cell classes typically used by DNAm-deconvolution algorithms are likely too broad to identify more subtle differences in dissection variability and potential stereological differences (van Haren et al. 2011; Nelson et al. 1998). While recent work has extended the number of cell populations that can be isolated by antibodies to separate neurons into their excitatory and inhibitory subclasses and oligodendrocytes from other glia (Kozlenkov et al. 2018), there are likely very few additional cell types that are possible to isolate using nuclear antibodies for DNAm samples. Researchers have therefore turned to using cell type-specific RNA microarray and sequencing datasets to adapt these reference-based deconvolution algorithms to homogenate RNA-seq samples (Avila Cobos et al. 2018; Newman et al. 2015; Mohammadi et al. 2017; Gong and Szustakowski 2013; Baron et al. 2016; Wang et al. 2020; Shen-Orr et al. 2010; Abbas et al. 2009; Wang et al. 2018; Burke et al. 2020; Collado-Torres et al. 2019). The majority of these studies have focused on tissues other than the brain, which can be freshly obtained and dissociated into individual cells for single cell RNA-seq (scRNAseq) or be sorted into specific cell populations using flow cytometry for cell type-specific expression profiling. For example, the popular CIBERSORT approach (Newman et al. 2015) was designed for blood gene expression microarray data, but has been adapted to RNA-seq datasets in other tissues. Several of the above algorithms have been designed, adapted or implemented for brain tissue, including linear regression followed by quadratic programming using the Houseman algorithm (Houseman et al. 2012; Collado-Torres et al. 2019; Burke et al. 2020), NNLS (Wang et al. 2018), the support vector machine-based CIBERSORT (Fromer et al. 2016; Hoffman et al. 2017), the empirical Bayes method MIND (Wang et al. 2020), and MuSiC, which combines a recursive tree based approach with weighted NNLS for cell type proportion estimation (Wang et al. 2019).

However, few of these approaches have validated that the resulting composition estimates are accurate, i.e., are absolutely similar to the true underlying composition, particularly in brain tissue. No approach to our knowledge has quantified the consequences of parameter and algorithm choices when only non-ideal reference data is available (e.g., mismatched tissue type, species, sequencing protocol, etc.), which occurs in almost all applications. Many reference datasets have been constructed from purified cell type-specific RNA-seq data from mouse (Xu et al. 2014), or RNA-seq data from sorted or dissociated nuclei in humans (Lake et al. 2016, 2018; Velmeshev et al. 2019; Hodge et al. 2019; Mathys et al. 2019), and not whole cells, which are typically profiled in homogenate sequencing studies. Gene expression levels are 
also quantitative within individual cells (and not binary like in DNAm data) and the necessity of absolute expression levels for absolute composition quantification has largely been overlooked.

Here we directly evaluated the absolute accuracy of several popular RNA-seq-based deconvolution strategies using several different reference datasets including a bulk/homogenate dataset with paired DNAm and RNA-seq data from the nucleus accumbens (NAc) from 200+ deceased individuals (Markunas et al. 2019). We used the DNAm data to estimate absolute neuronal fractions for each sample and evaluated absolute RNA-based deconvolution accuracy, using DNAm based neuronal fractions as proxies for the true unobserved fractions, across a variety of scenarios. We first evaluated the effects of using deep single cell RNA-seq (scRNA-seq) from healthy fresh human tissue obtained from surgically resected temporal cortex (Darmanis et al. 2015). This dataset likely produces the most comparable RNA-seq profiles to frozen bulk postmortem tissue, since whole cells were profiled, and 90\% of RNA is cytosolic in the cortex (Jaffe et al. 2015). However, this dataset was derived from cells in a cortical brain region. We next produced snRNA-seq data from postmortem human NAc to use as a reference dataset, which results in potentially less comparable nuclear reference profiles but comes from a more comparable brain region. We lastly used cyclic-ouroboros single-molecule fluorescence in situ hybridization (osmFISH) imaging data from the somatosensory cortex region in mouse (Codeluppi et al. 2018) to derive important parameters in popular deconvolution algorithms. Together, our results demonstrate that many algorithms are not accurate, even when estimating only two cell classes (neurons and glia), and we offer several strategies to assess and improve accuracy that can be applied across multiple datasets and cell types.

\section{Results}

We motivate this work with a large human postmortem brain genomic dataset from the NAc, a brain region containing functionally distinct cell types critical in reward-processing and addiction (Koob and Volkow 2016; Nestler 2005). Genomic data from this region has been underrepresented in postmortem human brain sequencing studies, which have primarily focused on the frontal cortex (Jaffe et al. 2018; Wang et al. 2018; Gandal et al. 2018) but its underrepresentation allows us to more comprehensively evaluate the accuracy of cellular deconvolution using potentially imperfect and/ or mismatched reference datasets (described below). We dissected homogenate NAc tissue from the ventral striatum (anterior to the optic chiasm) across 223 adult donors and concurrently extracted DNA and RNA from the exact same tissue aliquot (see Methods), which allows for directly comparable cellular composition in each fraction. We profiled genome-wide DNAm with the Illumina Infinium MethylationEPIC microarray and performed reference-based deconvolution to estimate the fraction of neurons in each sample (see Methods). We have previously demonstrated the absolute accuracy of the Houseman deconvolution algorithm (Houseman et al. 2012) in postmortem human brain DNAm data (Guintivano et al. 2013; Amanda J. Price et al. 2019); here we found very high correlation $(\rho=-0.949$, Figure S1, Extended data (Sosina et al. 2021)) between the neuronal fraction and the first principal component (PC) of the entire DNAm profile (32.3\% of variance explained), which we have shown to be an accurate surrogate of composition in frontal cortex (Jaffe et al. 2016) and blood (Jaffe and Irizarry 2014). The corresponding RNA was sequenced using the Illumina sequencing with RiboZero Gold library preparations (see Methods). This "gold standard" dataset, therefore, has DNAmderived neuronal composition values and RNA-seq data from 223 samples to explore the accuracy and concordance of many popular cellular deconvolution algorithms.

\section{Mismatched reference datasets bias deconvolution}

We first assessed the accuracy and concordance of four reference-based deconvolution algorithms: Houseman, CIBERSORT, NNLS/MIND, and MuSiC for two cell populations - neurons and non-neurons/glia - in our NAc RNA-seq dataset using recommended default settings (see Methods). We initially used single-cell RNA-seq (scRNA-seq) data from the temporal cortex of eight adult donors obtained during surgical resection generated and described in Darmanis et al. (2015) as the cell type-specific reference profiles for these algorithms. Importantly, these reference data were generated from fresh tissue, which preserved the integrity of the cells and corresponding cytosolic RNA, the predominant fraction of total RNA from brain (Jaffe et al. 2015) profiled in homogenate tissue. Furthermore, these reference profiles provide coverage of entire transcripts (as opposed to only the 3 ' ends) using Fluidigm C1 sequencing. Therefore, these expression profiles should be more comparable to bulk brain sequencing studies, with the caveat that the reference dataset was obtained from a different brain region (temporal cortex versus NAc and from living subjects as opposed to postmortem subjects).

We used measures of root mean square error (RMSE) to assess accuracy and squared Pearson correlation coefficients $\left(\mathrm{R}^{2}\right)$ to assess concordance for each algorithm's estimated neuronal fraction compared to the DNAm-based neuronal fractions (Figure 1). RMSE quantifies the degree of bias, i.e., how much our cell type estimates (RNA composition estimates) deviate from the inferred absolute cell type fractions, with smaller values corresponding to the inferred cellular composition and RNA composition being more similar. $\mathrm{R}^{2}$ quantifies the amount of information our estimates contain about how the inferred absolute cell type fractions vary in the population being studied, i.e., how much inferred variability of the unobserved cell type fractions, across individuals, is captured by our composition estimates. This is important since 
A

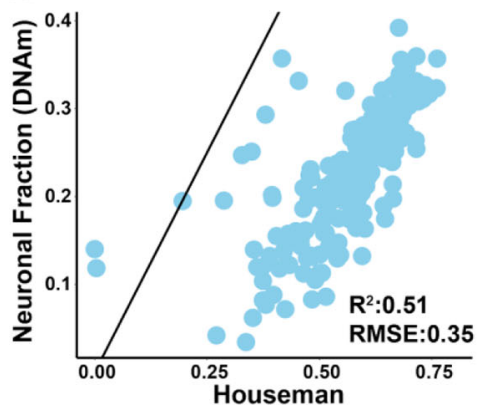

B

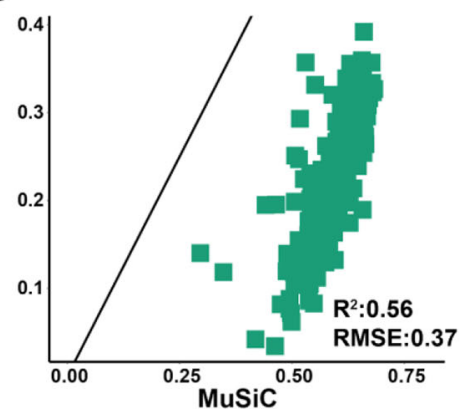

C

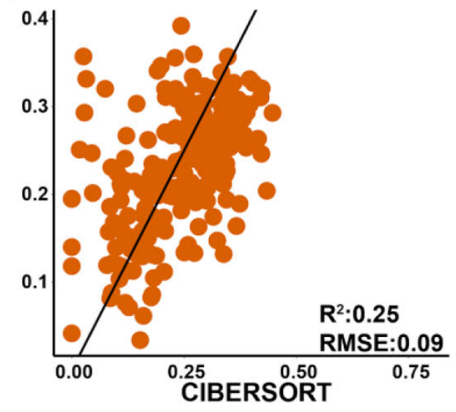

Figure 1. Deconvolution in bulk NAc data using gene expression profiles from the temporal cortex. Scatter plots showing the estimated neuronal proportions across the 223 individuals using the Houseman approach for DNAm reference vs neuronal proportions estimated using (A) the Houseman approach with scRNA reference, (B) MuSiC with default settings and scRNA reference data, and (C) CIBERSORT with scRNA reference data.

higher $\mathrm{R}^{2}$ values will lead to better elimination of cell-type composition effects in downstream analyses. Houseman (Figure 1A), MuSiC (Figure 1B), and NNLS produced concordant (high correlation; Houseman $\mathrm{R}^{2}=0.51,95 \% \mathrm{CI}[0.39$, 0.65]; $\mathrm{MuSiC} \mathrm{R}^{2}=0.56,95 \%$ CI [0.43, 0.69]; NNLS R ${ }^{2}=0.54,95 \%$ CI [0.32, 0.68]) but biased (high RMSE, $\geq 0.35$ ) neuronal fraction estimates. CIBERSORT produced more discordant (moderate correlation; $\mathrm{R}^{2}=0.25,95 \% \mathrm{CI}[0.15$, 0.38]) neuronal fraction estimates (Figure 1C), but with less bias (low RSME, 0.09). We found that CIBERSORT, compared to either MuSiC or the Houseman RNA approach, was the most accurate. However, its estimates had the lowest correlation $\left(\mathrm{R}^{2}\right)$ with estimates based on DNAm data. In comparing the $\mathrm{R}^{2}$ metric across the three approaches, we found that $\mathrm{MuSiC}$ had the highest correlation. Hence, it provides the most information about the inferred variability of the cell type proportions based on DNAm data. However, its estimates showed the greatest divergence, relative to other results, when compared to the inferred estimates from DNAm. These results suggest that all four of these approaches overestimated the inferred proportion of neurons in bulk brain tissue, even under the simplest application to deconvoluting two distinct cell populations. However, it was unclear how much algorithm parameters and reference dataset differences (in regard to technology and brain region) contributed to the performance of these methods.

\section{Methods for reducing bias in cellular deconvolution}

Many of the above deconvolution strategies have several parameters whose adjustment could reduce the observed bias (i.e. maximize accuracy) and increase the concordance between these neuronal fractions. The MuSiC algorithm particularly has an interpretable "cell size" (see Methods) parameter used in the deconvolution process. Different cell types could have more or less absolute RNA abundance, for example if they were larger or smaller, or if they were more or less transcriptionally active. We hypothesized that the overestimation of neuronal fractions resulted from neurons being larger and more transcriptionally active. However, this "cell size" parameter, regularly defined by the algorithm as the average expression level for a given cell type summed across genes, is estimated directly from the reference cell typespecific RNA-seq profiles by default (see Methods). However, some scRNA-seq (or snRNA-seq) library preparation and sequencing strategies, like the Fluidigm $\mathrm{C} 1$ system, may normalize cDNA libraries to the same concentration prior to sequencing, which will remove potential variability in RNA abundances across cell types. We therefore sought to use external data to better estimate these cell size parameters (Table 1) and assessed the resulting effects on cellular deconvolution accuracy.

First, we used external ouroboros single-molecule fluorescence in situ hybridization (osmFISH) data from mouse somatosensory cortex (Codeluppi et al. 2018) to construct two different types of cell size parameters for the MuSiC

Table 1. Cell sizes used for deconvolution.

\begin{tabular}{|c|c|c|c|c|c|c|}
\hline Cell type & $\begin{array}{l}\text { NAc } 50 \\
\text { genes } \\
\text { (UMIs) }\end{array}$ & $\begin{array}{l}\text { NAc } 25 \\
\text { genes } \\
\text { (UMIs) }\end{array}$ & $\begin{array}{l}\text { NAc all } \\
\text { genes } \\
\text { (UMIs) }\end{array}$ & $\begin{array}{l}\text { Temporal cortex, } \\
\text { Darmanis et al. } \\
\text { (Counts) }\end{array}$ & $\begin{array}{l}\text { osmFISH } \\
\text { cell Area } \\
\left(\mu \mathrm{m}^{2}\right)\end{array}$ & $\begin{array}{l}\text { osmFISH } \\
\text { nRNA } \\
\text { (intensity) }\end{array}$ \\
\hline Neuronal & 4513.58 & 2793.54 & 29884.65 & 18924.66 & 122.96 & 198.86 \\
\hline $\begin{array}{l}\text { Neuronal/ } \\
\text { glial ratio }\end{array}$ & 6.35 & 6.16 & 5.19 & 1.47 & 1.35 & 1.1 \\
\hline
\end{tabular}

UMI, unique molecular identifier, counts $=\log 2(\mathrm{cpm}+0.5)$, intensity $=$ sum of probe intensities across 24,048 genes . 
algorithm (as data from NAc did not exist). We extracted the estimates of both cell size (via their provided segmentations) and total RNA abundance (via the sum of all gene fluorescence signal) aggregated across neuronal and non-neuronal cell types. We subsequently utilized these estimates as proxies for cell size in human RNA-seq data when deconvoluting neuronal fractions. In these data, comparing neurons to non-neurons, neurons were both larger (123 vs $91 \mu \mathrm{m}^{2}, 95 \% \mathrm{CI}$ [28 $\left.\left.\mu \mathrm{m}^{2}, 36 \mu^{2}\right]\right)$ and had more total RNA (199 vs 180 intensity, 95\% CI [11, 25]) as we observed in the estimated cell size in the MuSiC algorithm using the Darmanis dataset (18,925 vs 12,880 normalized counts). We did not observe any improvement in the concordance (osmFISH cell area $\mathrm{R}^{2}=0.55,95 \% \mathrm{CI}[0.43,0.69]$; osmFISH totalRNA $\mathrm{R}^{2}=0.54$, $95 \% \mathrm{CI}[0.42,0.68]$ ) or accuracy (osmFISH cell area RMSE $=0.39$; osmFISH totalRNA RMSE $=0.43$ ) of the estimated cell type fractions when we compared our results from default settings to those based on applying cell size proxies using mouse data (Figure S2 A-B, Extended data (Sosina et al. 2021)). These results may not be particularly surprising, given the numerous differences between mouse and human morphology, and the different brain regions profiled.

We then generated snRNA-seq dataset from two postmortem NAc donors and 4,169 total nuclei to produce more comparable cell type-specific cell size (see Methods) parameters and reference expression profiles (see Methods) (Tran et al. 2020). First, we used the NAc reference dataset at the single nucleus level and ran the MuSiC algorithm with default settings, which used both NAc-based cell sizes and expression profiles, to deconvolute neuronal fractions (Figure 2A). We confirmed that, on average, neurons had more total RNA than non-neurons using this NAc snRNA-seq dataset (103 vs. 72 unique molecular identifiers [UMIs] per gene). Furthermore, while there was a high correlation among neuron-specific gene expression effects across the NAc and temporal cortex (Darmanis et al. 2015) reference profile datasets, we observed genes with different magnitudes of effects based on differential expression results between neuronal and non-neuronal cell types (Figure S3, Extended data (Sosina et al. 2021)). When using both the NAc-based cell size and gene expression reference profiles, we observed a substantial improvement in both the concordance and the RMSE for the estimated neuronal fractions compared to using the temporal cortex dataset only. However, the estimates were still biased, and this bias increased as the neuronal fraction across individuals increased, suggesting that the NAcbased cell sizes together with the estimated abundance may be incorrectly characterizing the true underlying neuronal expression level for these individuals. Eliminating the cell size parameter resulted in similarly reduced concordances in both the temporal cortex and the NAc reference datasets, but increased accuracy only using the NAc reference dataset (Table 2). This implies that the underlying broad cellular composition was well captured by the gene "abundance" information for a matched brain region.

We then combined different estimates of cell size parameters (NAc snRNA-seq versus osmFISH) and gene expression reference profiles (NAc snRNA-seq versus temporal cortex scRNA-seq) and assessed the effects on deconvolution accuracy in bulk NAc RNA-seq data. When running MuSiC using the estimates of cell size based on osmFISH data with the NAc expression reference profiles, we observed further improvements in the bias of the estimated cell type fractions but saw a minimal difference in the concordance (Figure 2B and C). Surprisingly, when we used only the Darmanis cell type-specific expression levels, the best (least biased and most concordant) deconvolution results were produced using cell sizes estimated from NAc snRNA-seq data, with improvements in both the concordance and the RMSE (Figure 3A). Specifically, when we compared the $\mathrm{R}^{2}$ and the RMSE estimates to those observed under the default setting for the Darmanis reference with the mismatched brain region, we see a small (6\% relative change, $95 \%$ CI $[0.18 \%, 11.67 \%])$
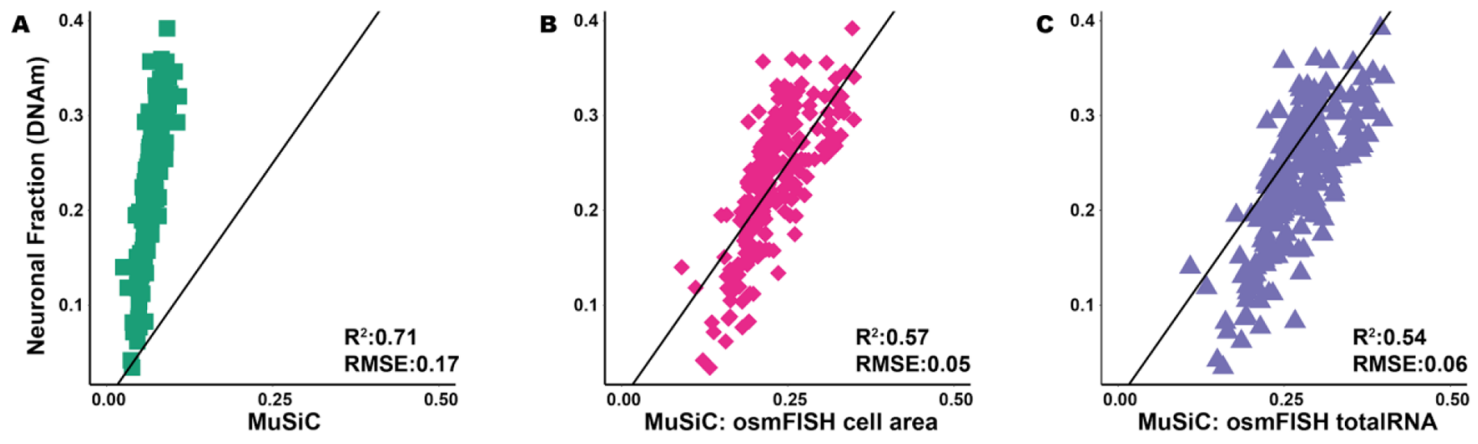

Figure 2. Deconvolution in bulk NAc data based on a single nucleus RNA-seq (snRNA-seq) reference dataset from the same brain region. Scatter plots comparing the estimated neuronal proportion obtained for each individual using the Houseman approach with DNAm reference dataset vs neuronal proportions obtained using (A) MuSiC with default settings and a snRNA-seq NAc reference dataset, (B) MuSiC based on a snRNA-seq NAc reference dataset with cell sizes for each cell type estimated using osmFISH cell area (mouse), and (C) MuSiC based on a snRNA-seq NAc reference dataset with cell sizes for each cell type estimated using osmFISH total RNA abundance (mouse) per cell type. 
Table 2. Bias and concordance results for deconvolution of bulk NAc data using each cell size and gene expression reference dataset.

\begin{tabular}{|c|c|c|c|c|c|}
\hline \multirow[t]{3}{*}{ Method } & \multirow[t]{3}{*}{ Cell size } & \multicolumn{4}{|c|}{ Reference dataset } \\
\hline & & \multicolumn{2}{|c|}{$\begin{array}{l}\text { scRNA-seq in temporal } \\
\text { cortex (Darmanis et al.) }\end{array}$} & \multicolumn{2}{|c|}{ snRNA-seq in NAc } \\
\hline & & $\begin{array}{l}\text { Concordance } \\
\left(R^{2}\right)\end{array}$ & $\begin{array}{l}\text { Accuracy } \\
\text { (RMSE) }\end{array}$ & $\begin{array}{l}\text { Concordance } \\
\left(\mathbf{R}^{2}\right)\end{array}$ & $\begin{array}{l}\text { Accuracy } \\
\text { (RMSE) }\end{array}$ \\
\hline \multirow[t]{7}{*}{ MuSiC } & None & 0.54 & 0.45 & 0.54 & 0.08 \\
\hline & $\begin{array}{l}\text { scRNA-seq in temporal cortex } \\
\text { (Darmanis et al.) }\end{array}$ & 0.56 & 0.37 & 0.58 & 0.05 \\
\hline & snRNA-seq in NAc & 0.59 & 0.08 & 0.71 & 0.17 \\
\hline & snRNA-seq in NAc (Top 25 genes) & 0.59 & 0.06 & 0.71 & 0.18 \\
\hline & snRNA-seq in NAc (Top 50 genes) & 0.59 & 0.05 & 0.72 & 0.18 \\
\hline & osmFISh-Cell area & 0.55 & 0.38 & 0.57 & 0.05 \\
\hline & osmFISh-Total RNA & 0.54 & 0.43 & 0.54 & 0.06 \\
\hline CIBERSORT & N/A & 0.25 & 0.09 & N/A & N/A \\
\hline NNLS & N/A & 0.54 & 0.40 & 0.72 & 0.22 \\
\hline
\end{tabular}
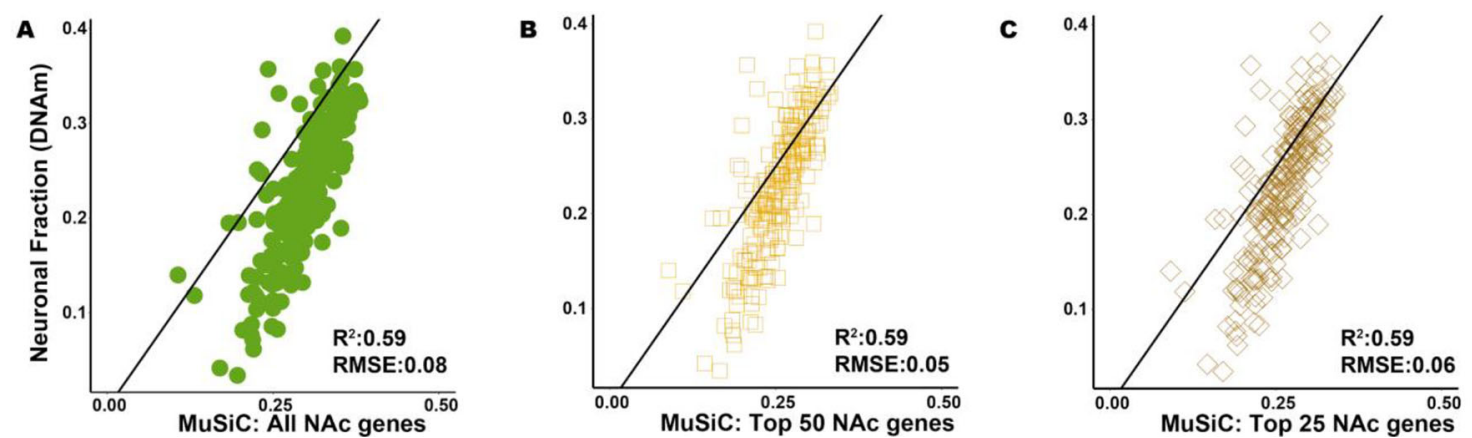

Figure 3. Deconvolution in bulk NAc data using gene expression profiles from the temporal cortex and different estimates of cell size. Scatter plots comparing the neuronal fraction estimated for each individual using DNAm data and the Houseman method vs neuronal fractions based on scRNA-seq data and estimated using MuSiC with (A) cell-size estimated using all genes expressed in the NAc snRNA-seq reference dataset, (B) cell-size estimated using the top 50 cell type discriminating genes in the NAc snRNA-seq reference dataset, and (C) cell-size estimated using the top 25 cell type discriminating genes in the NAc snRNA-seq reference dataset.

increase for the concordance and a substantial (78\% relative change, 95\% CI [77\%, 79\%]) decrease for the RMSE. We further refined the NAc cell size estimates using sets of the top 25 and 50 cell type discriminating genes (see Methods), which slightly improved our estimates of the absolute cell type fractions (Figure 3B and C). Both the concordance (7\% relative change, $95 \% \mathrm{CI}[0.16 \%, 12.74 \%])$ and RMSE (86\% relative change, 95\% CI [84\%, 87\%]) improved even more when compared to the default approach using a mismatched reference dataset. Across all approaches, the most accurate (least biased) result occurred when we used cell sizes estimated from Darmanis scRNA-seq data and gene expression from NAc snRNA-seq data, while the most concordant results were observed when we used NAc snRNA-seq data exclusively.

In summary, when we used a region-matched appropriate dataset - NAc snRNA-seq data - as the reference, or to derive estimates of the cell size, we observed that estimates of the cell type proportions generally improved (Table 2, Figure 2A and Figure 3A-C). In settings where we had a mismatched reference dataset (e.g., mismatched on brain region or species), incorporating estimated cell sizes obtained from the matched brain region (NAc) provided the best result in metrics for both concordance and accuracy, and we slightly improved these metrics when we refined the gene sets used to estimate the cell sizes. 


\section{Discussion}

Statistical deconvolution strategies have emerged over the past decade to estimate the proportion of various cell populations in homogenate tissue sources like blood and brain from both gene expression and DNAm data. Our results together suggest that many existing RNA deconvolution algorithms estimate the RNA composition of homogenate tissue, e.g. the amount of RNA attributable to each cell type, and not the cellular composition, which relates to the underlying fraction of cells. This was evident by the consistent overestimation of larger and more transcriptionally active neuronal cells. We have identified that incorporating cell size parameters into RNA-based deconvolution algorithms can successfully recover cellular fractions in homogenate brain RNA-seq data. We have lastly shown that using both cell sizes and cell type-specific gene expression profiles from brain regions other than the target/user-provided bulk tissue RNA-seq dataset consistently resulted in overestimating neuronal fractions. We have developed an extension of the MuSiC framework (Wang et al. 2019) that allows for the incorporation of independent cell size estimates, and have further provided cell size estimates for human brain (shown in Table 1) as a part of the package: https://github.com/ xuranw/MuSiC.

Characterizing cellular heterogeneity is especially important in human brain, where the underlying cell types can have diverse functions and disease associations that could be missed in studies of bulk tissue (Michels et al. 2013). Here we show that RNA-based deconvolution for just two cell populations - neurons and non-neurons - largely fails to estimate the underlying cellular composition of bulk human brain tissue across a variety of algorithms and strategies. We quantified the diverse range of neuronal fractions estimated by several popular algorithms to better understand the effects of reference cell type-specific expression profiles and differences in cell size and/or activity profiles on deconvolution. We specifically examined the common scenario of performing RNA deconvolution using cell type-specific reference datasets that can be fundamentally different from user-provided homogenate tissue target datasets, for example differing in profiled brain region, sequencing technology and/or cellular compartment. These problems are likely magnified in human brain tissue compared to suspended cells like blood, where deconvolution strategies are more easily validated against true cell fractions obtained by routine complete cell counts (Newman et al. 2015). We also note that while the effect of replacing the cell sizes is a general shift of all the points in the scatter plot which in turn changes the RMSE estimates, our goal in this paper is way to develop an approach to estimate the right shift needed to correct the biased estimates. Our results show that using the same dataset set to estimate both cell-size and abundance can lead to biased estimates. We lastly emphasize caution when performing RNA-based deconvolution using many cell types (i.e., more finely partitioned cell classes) without having the ability to validate cell counts on at least a subset of samples.

We therefore offer several recommendations for performing RNA-based deconvolution in bulk human brain gene expression data, particularly when aiming to identify cellular, and not RNA, composition.

1. Providing estimates of cell size for each reference cell type improves the concordance and reduces bias when performing RNA deconvolution to estimate cellular fractions. Biologically-motivated and valid external estimates of cell-size improve the accuracy of the estimated cell type fractions, even when gene expression profiles for reference cell populations are obtained from other brain regions (Figure 3). The exact biological interpretation of these estimated cell sizes, particularly when estimated across species, is arguably unclear, but likely relates to correcting for absolute RNA abundance and differences in transcriptional activity between cell populations. Regardless of the method used for deriving cell sizes, neurons consistently had more RNA than glia. We note that our recommended strategies for estimating cell size have only been assessed for broad classes of cell types, and further work is needed to validate extensions to more stratified subclasses of cells.

2. The concordance and bias improvements using full-length single cell sequencing from a different brain region (temporal cortex), rather than single nuclei RNA-seq from the target brain region (NAc) highlighted the importance of comparability between reference gene expression profiles and the homogenate tissue expression levels. While previous reports have identified high correlation between nuclear and cytosolic gene expression levels in both bulk (Amanda J. Price et al. 2019) and single cell (Bakken et al. 2018; Hodge et al. 2019) resolution, comparable absolute (and not relative) expression levels are seemingly important for the accuracy of these RNA-based cellular deconvolution algorithms. There further is an experimental design tradeoff between profiling more nuclei (1000s) using 3' technologies like 10x Genomics Chromium Single Cell Gene Expression compared to profiling fewer nuclei (or cells, 100s) using full-length sequencing technologies like SMART-seq if researchers wish to generate their own reference profiles.

3. Using reference cell type-specific expression profiles from comparable brain regions as the bulk RNA-seq target dataset is important and can especially greatly increase the concordance of these RNA deconvolution strategies with neuronal fractions. 
The choice of maximizing accuracy (by minimizing bias) versus increasing concordance in assessing these algorithms is an important consideration, particularly when generating custom expression reference profiles is prohibitive (Table 2). These two objectives largely relate to whether the goal of RNA deconvolution is to obtain correct estimates of absolute cell fractions (and maximize accuracy) or to identify/leverage differences in cell/RNA fractions (and maximize concordance) as a basis to obtain cell-type specific inference for a given gene. Estimation of RNA fractions (by maximizing concordance) may be sufficient to control for potential confounding due to composition differences between outcome groups (Michels et al. 2013). We note this can also be accomplished using "reference-free" deconvolution (Houseman et al. 2014) or through the estimation of potentially sparse principal components (Rahmani et al. 2016; Jaffe and Irizarry 2014) that control for relative differences in cellular composition. However, estimation of cellular fractions (and maximizing accuracy) is arguably more useful, both for assessing human brain tissue dissection during data generation and to identify cell type-specific effects when using these cellular fractions in downstream differential expression analyses (Zheng et al. 2018).

Together, our results demonstrate that many RNA deconvolution algorithms do not produce accurate cellular fractions when estimating only two cell classes (neurons and non-neurons). We offer several strategies and corresponding software to assess and improve accuracy that can be applied across multiple datasets and cell types.

\section{Methods}

Ethics statement

Brain donation protocols were approved by IRB and oversight bodies. Legal next-of-kin gave informed consent to brain donation according to protocols Maryland Department of Health MDHMH\# 12-24 (MD), National Institute of Mental Health [NIMH]\# 90-M-014 (for brain donations in DC and VA), and Western Institutional Review Board [WIRB] \# 1126332 (for brain donations in MD, WMU, UND), respectively. Subsequent research on human postmortem brain tissue - including the RNA sequencing performed here - is not classified as Human Subjects Research (since postmortem subjects are not classified as Human Subjects, per the Department of Health and Human Services in DHHS 45 CFR $\$ 46.102(\mathrm{e})(1):$.

\section{Bulk NAc data generation and processing}

Data generation and processing were described extensively in Markunas et al. (2019). Briefly, the nucleus accumbens (NAc) was dissected under visual guidance using a hand-held dental drill. Samples were obtained from the ventral striatum, anterior to the optic chiasm, at the level where the NAcforms a bridge between the putamen and the head of the caudate. DNA and RNA were concurrently extracted from dissected tissue using the Qiagen AllPrep DNA/RNA Mini Kit (Cat No./ID: 80204).

NAc DNA was profiled with the Infinium MethylationEPIC microarray (catalog \#: WG-317-1003) using the manufacturer's protocol. Raw idat files were processed and normalized using the minfi Bioconductor package (Aryee et al. 2014) using stratified quantile normalization (Touleimat and Tost 2012). Resulting neuronal fractions were estimated using the minfi estimate CellCounts function (Jaffe and Irizarry 2014) using sorted reference data from the DLPFC for neurons and non-neurons (Guintivano et al. 2013; Andrew E. Jaffe 2017) using the Houseman algorithm (Houseman et al. 2012).

NAc RNA was subjected to RNA-seq library preparations using the Illumina RiboZero Gold kits (catalog \#: 20020599) and sequenced using $2 \times 100$ bp paired end reads on an Illumina HiSeq 3000 .

\section{Reference datasets}

Darmanis: SRP057196 (Darmanis et al. 2015).

scRNA-seq data for 58,037 genes and 556 cells were obtained for brain samples across eight individuals, as described previously (Darmanis et al. 2015). We filtered this dataset by removing cells based on embryonic samples and retaining cells from one of the following five cell types: neuronal, oligodendrocyte progenitor cells (OPC), astrocytes, oligodendrocytes, and microglia. We also removed genes that had no expression for all cells in the reference dataset or did not show any expression in the bulk dataset (i.e., mean and variance zero). In total, we used 265 cells for this reference and 24,048 genes to estimate the cell type proportions for the 223 samples with bulk NAc data.

Single-nucleus RNA-seq data generation and processing in nucleus accumbens

We performed single-nucleus RNA-seq (snRNA-seq) on nucleus accumbens (NAc) tissue from two donors using $10 \times$ Genomics Single Cell Gene Expression V3 technology (PN-1000075) as previously described (Tran et al. 2020). Briefly, 
nuclei were isolated using a "Frankenstein" nuclei isolation protocol developed by Martelotto et al. for frozen tissues (2017; Lacar et al. 2016; Lake et al. 2016; Habib et al. 2016; Hu et al. 2017). Briefly, 40 mg of frozen NAc tissue was homogenized in chilled Nuclei EZ Lysis Buffer (MilliporeSigma) in a glass dounce with $\sim 15$ strokes per pestle. Homogenate was filtered using a $70 \mathrm{um}$-strainer mesh and centrifuged at $500 \times \mathrm{g}$ for 5 minutes at $4^{\circ} \mathrm{C}$ in a benchtop centrifuge. Nuclei were resuspended in the EZ lysis buffer, centrifuged again, and equilibrated to nuclei wash/resuspension buffer $(1 \times \mathrm{PBS}$, $1 \%$ BSA, 0.2 U/uL RNase Inhibitor). Nuclei were washed and centrifuged in this nuclei wash/resuspension buffer three times, before labeling with DAPI $(10 \mathrm{ug} / \mathrm{mL})$. The sample was then filtered through a $35 \mathrm{um}$-cell strainer and sorted on a BD FACS Aria II Flow Cytometer (Becton Dickinson) at the Johns Hopkins University Sidney Kimmel Comprehensive Cancer Center (SKCCC) Flow Cytometry Core. Gating criteria were hierarchically selected for whole, singlet nuclei (by forward/ side scatter), then for $\mathrm{G}_{0} / \mathrm{G}_{1}$ nuclei (by DAPI fluorescence). A null sort was additionally performed from the same preparation to ensure nuclei input was free of debris. Approximately 8,500 single nuclei were sorted directly into 25.1uL of reverse transcription reagents from the $10 \times$ Genomics Single Cell 3' Reagents kit (PN-1000075, without enzyme). Libraries were prepared according to manufacturer's instructions $(10 \times$ Genomics) and sequenced on the Next-seq (Illumina) at the Johns Hopkins University Transcriptomics and Deep Sequencing Core.

We processed the sequencing data with the $10 \times$ Genomics' Cell Ranger pipeline, aligning to the human reference genome GRCh38, with a reconfigured GTF such that intronic alignments were additionally counted given the nuclear context, to generate UMI/feature-barcode matrices. We used R package Seurat (Satija et al. 2015) for raw feature-barcode quality control, dimensionality reduction (PCA), choosing the top $30 \mathrm{PCs}$ as the optimal dimensions for clustering. We performed graph-based clustering with the default Louvain approach, taking a computed K-nearest neighbors graph as input, which were then annotated with well-established cell type markers for nuclear type identity (Mathys et al. 2019). We also used Seurat's implementation of non-linear dimensionality reduction techniques, t-SNE and UMAP, simply for visualization of the high-dimensional structure in the data, which complemented the clustering results (Figure S4, Extended data (Sosina et al. 2021)). With the five broad cell type annotations (neurons, oligodendrocytes, oligodendrocyte precursors, astrocytes, and microglia) of nuclear clusters, we identified unbiased cluster-driving genes (with Seurat's 'FindAllMarkers()' function, using the Wilcoxon rank-sum test), that were upregulated in each cell type/cluster, compared to all other nuclei. Using the same set of 24,048 genes, we have 4,169 high-quality nuclei in this reference, evenly distributed across donors. The top 50- and top 25-per-cell-type gene sets had 247 and 125 genes, respectively, which included many cell type marker genes used for annotation.

\section{Estimation procedures}

\section{HOUSEMAN}

This algorithm (Houseman et al. 2012) uses a linearly constrained quadratic optimization approach with additional nonnegative constraints on the parameters. The linear constraint does not require that the sum of all coefficients equal one. This allows the possibility of unknown cell types in case the specification is not comprehensive. It was implemented using the minfi R Bioconductor package (Aryee et al. 2014).

\section{MuSiC}

The MuSiC (Wang et al. 2019) approach models the relationship between the relative abundance of gene $\mathrm{g}$ in the bulk RNA-seq data and the mean expression level of the same gene in the reference dataset for a given individual. The relationship is provided below

$$
Y_{g} \propto \sum_{k=1}^{K} p^{k} S^{k} \theta_{g}^{k}
$$

Where $k=1, \ldots, K$ is the index of the cell types, $p^{k}$ is the proportion of cells from cell type $k$, and $\theta_{g}^{k}$ is the relative abundance for the $g$ th gene with respect to the kth cell type. $S^{k}$ is the cell size parameter and is defined as the average number of total mRNA molecules for cell type k. By default, $S^{k}$ is estimated automatically by MuSiC. For the deconvolution method comparisons that assessed cell size impact on neuronal cell type proportion estimation, $S^{k}$ was derived from one of multiple data sources (Table 1) using 1) default settings 2) osmFISH or 3) the average number of total mRNA molecules for cell type k using only the top 25 or 50 most discriminating genes per cell type. We defined "most discriminating" as genes with the smallest $\mathrm{p}$-values and fold change $>0.25$, relative to other cell types. All estimation was carried out using the MuSiC package in $\mathrm{R}$ v3.6.3. 


\section{CIBERSORT}

CIBERSORT uses a machine learning approach called nu-support vector regression (Newman et al. 2015; Schölkopf et al., 2000) and requires at least two input datasets to work. The first is a signature matrix that identifies the set of genes that are informative for the deconvolution procedure. The second is a bulk RNA-seq dataset to estimate cell type proportions.

The signature matrix depends on the tissue of interest. We generated a custom signature matrix. Using the Darmanis reference dataset, we generated both a reference sample file (gene-by-cell matrix) and a phenotype classes file (cell typeby-dummy variable identifying the cell type for each cell) and used the default setting (CIBERSORT) to obtain a custom signature gene expression matrix. The specified false discovery rate (FDR) threshold used to include genes in the signature matrix was 0.30 (i.e. $q=0.30$, default). Using this signature matrix, we then performed deconvolution on our bulk NAc RNA-seq data. As suggested in the documentation for CIBERSORT, we disabled quantile normalization for our RNA-seq data.

\section{NNLS/MIND}

This is a simple linear regression with non-negativity constraints on the parameter estimates. The estimated fractions are then the value of each parameter estimate divided by the sum of all parameter estimates across cell types. MIND uses NNLS to estimate cell type fractions.

\section{Data availability}

Underlying data

Gene Expression Omnibus: Genome-wide RNA-sequencing differences in nucleus accumbens of smokers vs. nonsmokers, Accession number GSE171936: https://identifiers.org/geo:GSE171936.

\section{Extended data}

Zenodo: Strategies for cellular deconvolution in human brain RNA sequencing data. https://doi.org/10.5281/zenodo. 4899937 (Sosina et al. 2021).

This project contains the following extended data:

- sosina_extended_sf1.docx (Figure S1, DNAm estimated neuronal fractions vs PC1)

- sosina_extended_sf2.docx (Figure S2, Deconvolution in bulk NAc data using gene expression profiles from the temporal cortex with cell size estimates derived using mouse samples)

- sosina_extended_sf3.docx (Figure S3, Neuronal enrichment of gene expression in scRNA-seq from temporal cortex and snRNA-seq from nucleus accumbens)

- sosina_extended_sf4.docx (Figure S4, t-distributed stochastic neighbor embedding (t-SNE) of single-nucleus RNA-seq data from the two postmortem NAc samples, representing the 4,169 high-quality nuclei after processing)

Data are available under the terms of the Creative Commons Attribution 4.0 International license (CC-BY 4.0).

Analysis code available from: https://github.com/ksosina/Strategies-for-cellular-deconvolution-in-human-brain-RNAsequencing-data.

Archived analysis code at time of publication: https://doi.org/10.5281/zenodo.5009842 (ksosina, 2021).

License: CC0 1.0 Universal.

\section{Acknowledgements}

The authors thank the authors of MuSiC; X. Wang, J. Park, K. Susztak, N.R. Zhang, and M. Li for their technical assistance and valuable insights. 
Abbas AR, Wolslegel K, Seshasayee D, et al.: Deconvolution of blood microarray data identifies cellular activation patterns in systemic lupus erythematosus. Plos One. 2009; 4(7): e6098.

PubMed Abstract | Publisher Full Text | Free Full Text

Jaffe AE, K ZA: FlowSorted.DLPFC.450k. Bioconductor. 2017.

Publisher Full Text

Aryee MJ, Jaffe AE, Corrada-Bravo $\mathrm{H}$, et al.: Minfi: a flexible and comprehensive Bioconductor package for the analysis of Infinium DNA methylation microarrays. Bioinformatics. 2014; 30(10): 1363-1369. PubMed Abstract | Publisher Full Text | Free Full Text

Avila Cobos F, Vandesompele J, Mestdagh P, et al.: Computational deconvolution of transcriptomics data from mixed cell populations. Bioinformatics. 2018; 34(11): 1969-1979.

PubMed Abstract | Publisher Full Text

Bakken TE, Hodge RD, Miller JA, et al.: Single-nucleus and single-cell transcriptomes compared in matched cortical cell types. Plos One. 2018; 13(12): e0209648.

PubMed Abstract | Publisher Full Text | Free Full Text

Baron M, Veres A, Wolock SL, et al.: A Single-Cell Transcriptomic Map of the Human and Mouse Pancreas Reveals Inter- and Intra-cell Population Structure. Cell Systems. 2016; 3(4): 346-360. e4. PubMed Abstract | Publisher Full Text | Free Full Text

Burke EE, Chenoweth JG, Shin JH, et al.: Dissecting transcriptomic signatures of neuronal differentiation and maturation using iPSCs. Nat Commun. 2020; 11(1): 462

PubMed Abstract | Publisher Full Text | Free Full Text

Codeluppi S, Borm LE, Zeisel A, et al.: Spatial organization of the somatosensory cortex revealed by osmFISH. Nat Methods. 2018; 15(11): 932-935

PubMed Abstract | Publisher Full Text

Collado-Torres L, Burke EE, Peterson A, et al.: Regional Heterogeneity in Gene Expression, Regulation, and Coherence in the Frontal Cortex and Hippocampus across Development and Schizophrenia. Neuron. 2019; 103(2): 203-216. e8.

PubMed Abstract | Publisher Full Text | Free Full Text

Darmanis S, Sloan SA, Zhang Y, et al.: A survey of human brain transcriptome diversity at the single cell level. Proc Natl Acad Sci U SA. 2015; 112(23): 7285-7290.

PubMed Abstract | Publisher Full Text | Free Full Text

Fromer M, Roussos P, Sieberts SK, et al.: Gene expression elucidates functional impact of polygenic risk for schizophrenia. Nat Neurosci.

2016; 19(11): 1442-1453.

PubMed Abstract | Publisher Full Text | Free Full Text

Gandal MJ, Zhang P, Hadjimichael E, et al.: Transcriptome-wide isoformlevel dysregulation in ASD, schizophrenia, and bipolar disorder.

Science. 2018; 362(6420)

PubMed Abstract | Publisher Full Text | Free Full Text

Gong T, Szustakowski JD: DeconRNASeq: a statistical framework for deconvolution of heterogeneous tissue samples based on mRNA-Seq data. Bioinformatics. 2013; 29(8): 1083-1085.

PubMed Abstract | Publisher Full Text

Guintivano J, Aryee MJ, Kaminsky ZA: A cell epigenotype specific model for the correction of brain cellular heterogeneity bias and its

application to age, brain region and major depression. Epigenetics.

2013; 8(3): 290-302.

PubMed Abstract | Publisher Full Text | Free Full Text

Habib N, Avraham-Davidi I, Basu A, et al.: Massively parallel singlenucleus RNA-seq with DroNc-seq. Nat Methods. 2017; 14(10): 955-958. PubMed Abstract | Publisher Full Text | Free Full Text

Habib N, Li Y, Heidenreich M, et al.: Div-Seq: Single-nucleus RNA-Seq reveals dynamics of rare adult newborn neurons. Science. 2016; 353(6302): 925-928.

PubMed Abstract | Publisher Full Text | Free Full Text

Hodge RD, Bakken TE, Miller JA, et al.: Conserved cell types with divergent features in human versus mouse cortex. Nature. 2019; 573(7772): 61-68

PubMed Abstract | Publisher Full Text | Free Full Text

Hoffman GE, Hartley BJ, Flaherty E, et al.: Transcriptional signatures of schizophrenia in hiPSC-derived NPCs and neurons are concordant

with post-mortem adult brains. Nat Commun. 2017; 8(1): 2225.

PubMed Abstract | Publisher Full Text | Free Full Text

Houseman EA, Accomando WP, Koestler DC, et al.: DNA methylation arrays as surrogate measures of cell mixture distribution. $B M C$ Bioinformatics. 2012: 13: 86 .

PubMed Abstract | Publisher Full Text | Free Full Text

Houseman EA, Molitor J, Marsit C]: Reference-free cell mixture adjustments in analysis of DNA methylation data. Bioinformatics. 2014; 30(10): 1431-1439.

PubMed Abstract | Publisher Full Text | Free Full Text
Hu P, Fabyanic E, Kwon DY, et al.: Dissecting Cell-Type Composition and Activity-Dependent Transcriptional State in Mammalian Brains by Massively Parallel Single-Nucleus RNA-Seq. Mol Cell. 2017; 68(5),

pp. 1006-1015. e7.

PubMed Abstract | Publisher Full Text | Free Full Text

Jaffe AE, Gao Y, Deep-Soboslay A, et al.: Mapping DNA methylation across development, genotype and schizophrenia in the human frontal cortex. Nat Neurosc. 2016; 19(1): 40-47.

PubMed Abstract | Publisher Full Text | Free Full Text

Jaffe AE, Irizarry RA: Accounting for cellular heterogeneity is critical in epigenome-wide association studies. Genome Biol. 2014; 15(2): R31. PubMed Abstract | Publisher Full Text | Free Full Text

Jaffe AE, Shin J, Collado-Torres L, et al.: Developmental regulation of human cortex transcription and its clinical relevance at single base resolution. Nat Neurosci. 2015; 18(1): 154-161.

PubMed Abstract | Publisher Full Text | Free Full Text

Jaffe $A E$, Straub RE, Shin JH, et al.: Developmental and genetic regulation of the human cortex transcriptome illuminate schizophrenia

pathogenesis. Nat Neurosci. 2018; 21(8): 1117-1125.

PubMed Abstract | Publisher Full Text | Free Full Text

Koob GF, Volkow ND: Neurobiology of addiction: a neurocircuitry analysis. Lancet. Psychiatry. 2016; 3(8): 760-773.

PubMed Abstract | Publisher Full Text | Free Full Text

Kozlenkov A, Li J, Apontes P, et al.: A unique role for DNA (hydroxy) methylation in epigenetic regulation of human inhibitory neurons.

Sci Adv. 2018; 4(9): eaau6190.

PubMed Abstract | Publisher Full Text | Free Full Text

ksosina: ksosina/Strategies-for-cellular-deconvolution-in-humanbrain-RNA-sequencing-data: Initial release (Version v1.0.0). Zenodo. 2021, June 22.

Publisher Full Tex

Lacar B, Linker SB, Jaeger BN, et al.: Nuclear RNA-seq of single neurons reveals molecular signatures of activation. Nat Commun. 2016; 7:

11022

PubMed Abstract | Publisher Full Text | Free Full Text

Lake BB, Ai R, Kaeser GE, et al.: Neuronal subtypes and diversity revealed by single-nucleus RNA sequencing of the human brain.

Science. 2016; 352(6293): 1586-1590.

PubMed Abstract | Publisher Full Text | Free Full Text

Lake BB, Chen S, Sos BC, et al.: Integrative single-cell analysis of transcriptional and epigenetic states in the human adult brain. Nat Biotechnol. 2018; 36(1): 70-80.

PubMed Abstract | Publisher Full Text | Free Full Text

Markunas CA, Semick SA, Quach BC, et al.: Genome-wide DNA methylation differences in nucleus accumbens of smokers

vs. nonsmokers. BioRxiv. 2019.

PubMed Abstract | Publisher Full Text |Free Full Text

Mathys H, Davila-Velderrain J, Peng Z, et al.: Single-cell transcriptomic analysis of Alzheimer's disease. Nature. 2019; 570(7761): 332-337.

PubMed Abstract | Publisher Full Text | Free Full Text

Michels KB, Binder AM, Dedeurwaerder S, et al.: Recommendations fo the design and analysis of epigenome-wide association studies. $\mathrm{Nat}$ Methods. 2013; 10(10): 949-955.

PubMed Abstract | Publisher Full Text

Mohammadi S, Zuckerman N, Goldsmith A, et al.: A critical survey of deconvolution methods for separating cell types in complex tissues. Proc IEEE. 2017; 105(2): 340-366.

Publisher Full Text

Montaño CM, Irizarry RA, Kaufmann WE, et al.: Measuring cell-type specific differential methylation in human brain tissue. Genome Biol. 2013; 14(8): R94.

PubMed Abstract | Publisher Full Text | Free Full Text

Nelson MD, Saykin AJ, Flashman LA, et al.: Hippocampal volume reduction in schizophrenia as assessed by magnetic resonance imaging: a meta-analytic study. Arch Gen Psychiatry. 1998; 55(5): 433-440.

PubMed Abstract | Publisher Full Text

Nestler Ej: Is there a common molecular pathway for addiction? Nat Neurosci. 2005; 8(11): 1445-1449.

PubMed Abstract | Publisher Full Text

Newman AM, Liu CL, Green MR, et al.: Robust enumeration of cell subsets from tissue expression profiles. Nat Methods. 2015; 12(5): 453-457.

PubMed Abstract | Publisher Full Text | Free Full Text

Price AJ, Collado-Torres L, Ivanov NA, et al.: Divergent neuronal DNA methylation patterns across human cortical development reveal critical periods and a unique role of $\mathrm{CpH}$ methylation. Genome Biol. 2019; 20(1): 196.

PubMed Abstract | Publisher Full Text | Free Full Text 
Price AJ, Hwang T, Tao R, et al.: Characterizing the nuclear and cytoplasmic transcriptomes in developing and mature human cortex uncovers new insight into psychiatric disease gene regulation. BioRxiv. 2019.

Publisher Full Text

Rahmani E, Zaitlen N, Baran Y, et al.: Sparse PCA corrects for cell type heterogeneity in epigenome-wide association studies. Nat Methods. 2016; 13(5): 443-445.

PubMed Abstract | Publisher Full Text | Free Full Text

Satija R, Farrell JA, Gennert D, et al.: Spatial reconstruction of single-cell gene expression data. Nat Biotechnol. 2015; 33(5): 495-502.

PubMed Abstract | Publisher Full Text | Free Full Text

Shen-Orr SS, Tibshirani R, Khatri P, et al.: Cell type-specific gene expression differences in complex tissues. Nat Methods. 2010; 7(4): 287-289.

PubMed Abstract | Publisher Full Text | Free Full Text

Sosina OA, Leek JT, Jaffe AE: Strategies for cellular deconvolution in human brain RNA sequencing data (Version v1). Zenodo. 2021, June 4. Publisher Full Text

Touleimat N, Tost J: Complete pipeline for Infinium( (®) Human Methylation 450K BeadChip data processing using subset quantile normalization for accurate DNA methylation estimation. Epigenomics. 2012; 4(3): 325-341.

PubMed Abstract | Publisher Full Text

Tran MN, Maynard KR, Spangler A, et al.: Single-nucleus transcriptome analysis reveals cell type-specific molecular signatures across reward

circuitry in the human brain. BioRxiv. 2020.

Publisher Full Text van Haren NEM, Schnack HG, Cahn W, et al.: Changes in cortical thickness during the course of illness in schizophrenia. Arch Gen Psychiatry. 2011; 68(9), pp. 871-880.

PubMed Abstract | Publisher Full Text

Velmeshev D, Schirmer L, Jung D, et al.: Single-cell genomics identifies cell type-specific molecular changes in autism. Science. 2019; 364(6441): 685-689.

PubMed Abstract | Publisher Full Text | Free Full Text

Wang D, Liu S, Warrell J, et al.: Comprehensive functional genomic resource and integrative model for the human brain. Science. 2018, 362(6420).

PubMed Abstract | Publisher Full Text | Free Full Text

Wang J, Devlin B, Roeder K: Using multiple measurements of tissue to estimate subject- and cell-type-specific gene expression.

Bioinformatics. 2020; 36(3): 782-788.

PubMed Abstract | Publisher Full Text | Free Full Text

Wang X, Park J, Susztak K, et al.: Bulk tissue cell type deconvolution with multi-subject single-cell expression reference. Nat Commun. 2019; 10(1): 380.

PubMed Abstract | Publisher Full Text | Free Full Text

Xu X, Wells AB, O'Brien DR, et al.: Cell type-specific expression analysis to identify putative cellular mechanisms for neurogenetic disorders.

J Neurosci. 2014; 34(4): 1420-1431.

PubMed Abstract | Publisher Full Text | Free Full Text

Zheng SC, Breeze CE, Beck S, et al.: Identification of differentially methylated cell types in epigenome-wide association studies.

Nat Methods. 2018; 15(12): 1059-1066.

PubMed Abstract | Publisher Full Text | Free Full Text 


\section{Open Peer Review}

\section{Current Peer Review Status:}

\section{Version 1}

Reviewer Report 22 August 2022

https://doi.org/10.5256/f1000research.53947.r146615

(C) 2022 Bhattacharya A. This is an open access peer review report distributed under the terms of the Creative Commons Attribution License, which permits unrestricted use, distribution, and reproduction in any medium, provided the original work is properly cited.

\section{Arjun Bhattacharya}

Department of Pathology and Laboratory Medicine, David Geffen School of Medicine, University of California, Los Angeles, Los Angeles, CA, USA

In this manuscript, the authors present a thorough benchmarking of cell-type deconvolution methods and motivate new datasets to benchmark these methods. Their findings emphasize a very important fact: that cell-type deconvolutions methods are often not suitable to estimate celltype proportions, but rather the proportion of total RNA produced by different cell types.

Generally, I have no major comments/critiques of the paper. I believe this is a very thorough examination of cell-type deconvolution methods, with a specific conclusion that is important when deconvoluted proportions are included in the downstream analyses. I would suggest a citation for the linseed method (Zaitsev et al., 2019 ${ }^{1}$ ) would be helpful, as this question of cell size vs. RNA produced is discussed in this methods paper. Most important, the addition of the "gold standard" dataset is very important, as many existing datasets that are used to benchmark these methods are outdated.

\section{References}

1. Zaitsev K, Bambouskova M, Swain A, Artyomov M: Complete deconvolution of cellular mixtures based on linearity of transcriptional signatures. Nature Communications. 2019; 10 (1). Publisher Full Text

Is the rationale for developing the new method (or application) clearly explained? Yes

Is the description of the method technically sound?

Yes

Are sufficient details provided to allow replication of the method development and its use by others? 
Yes

If any results are presented, are all the source data underlying the results available to ensure full reproducibility?

Yes

Are the conclusions about the method and its performance adequately supported by the findings presented in the article?

Yes

Competing Interests: No competing interests were disclosed.

Reviewer Expertise: Statistical genetics, bioinformatics, transcriptomics, brain traits, cancer

I confirm that I have read this submission and believe that I have an appropriate level of expertise to confirm that it is of an acceptable scientific standard.

Reviewer Report 02 February 2022

https://doi.org/10.5256/f1000research.53947.r99217

(C) 2022 Roeder $\mathrm{K}$ et al. This is an open access peer review report distributed under the terms of the Creative Commons Attribution License, which permits unrestricted use, distribution, and reproduction in any medium, provided the original work is properly cited.

\section{Kathryn Roeder}

Department of Statistics and Data Science, Carnegie Mellon University, Pittsburgh, PA, USA Jiebiao Wang

Department of Biostatistics, University of Pittsburgh, Pittsburgh, PA, USA

Sosina et al. compared the performance of methods to perform cellular deconvolution of bulk gene expression from brains. They question the performance of popular methods when they use reference samples from mismatched brain regions and ignore cell size. Differential cell size leads to a fundamental difference between the fraction of cells of each type, versus the fraction of transcripts originating from each cell type. The authors seem to take the view that the former quantity is generally more appropriate. In their study they use paired bulk DNA methylation (DNAm) and gene expression data from the same subjects, so that they can estimate cell fraction fairly accurately from the DNAm. This quantity is used as ground truth. The paper presents its message clearly and provides a comprehensive inquiry of the question at hand. Portions of the text would benefit from revision to improve the writing style. Below are some comments that may improve the manuscript:

1. In the discussion, the authors say "We also note that while the effect of replacing the cell sizes is a general shift of all the points in the scatter plot which in turn changes the RMSE estimates, our goal in this paper is way to develop an approach to estimate the right shift needed to correct the biased estimates". This statement is awkwardly phrased, and this is troubling because this is a key point of the paper. Apparently, their point is that RMSE is 
affected by location shift, and using cell size to correct for this location shift may not help much in downstream analyses.

2. The authors need to more clearly differentiate between downstream applications where the true cell type fraction is required and others where it is actually better to estimate the fraction of transcripts originating from each cell type. The authors mention that "Estimation of RNA fractions may be sufficient to control for potential confounding due to composition differences between outcome groups". But they should go further to note that for some downstream analyses that aim to estimate cell-type-specific expression (e.g., MIND and bMIND) (Bakken et al. $\left(2018^{1}\right)$ ) it does not make much difference to estimate the transcript or cellular fraction. The authors do say, "However, estimation of cellular fractions (and maximizing accuracy) is arguably more useful, both for assessing human brain tissue dissection during data generation and to identify cell type-specific effects when using these cellular fractions in downstream differential expression analyses (Zheng et al. 2018)." This statement relies very heavily on the cited reference, but we find very little difference between controlling for cell fractions versus RNA fractions in that report. Indeed the difference amounts to adding an interaction of fractions and outcome to the regression, and adding/subtracting a constant for the fractions may not matter much to the key inferences. Simulations/analyses are needed to convince readers that RMSE is an important metric, and using cell size to correct for the mean fraction matters in downstream analysis.

3. The authors used paired DNAm and RNA-seq data, and the Houseman's approach estimated neuronal fraction as the gold standard. Patrick et al.(2020²) measured cellular fractions from 70 individuals. With DNAm measured from those individuals and the reference and Houseman's approach implemented in the minfi package, we observed R2 = 0.008 and RMSE $=0.08$. This contradicts the claim that they have a "gold standard", and some justification is required.

4. Bakken et al. $\left(2018^{1}\right)$ used well-matched snRNA-seq and scRNA-seq datasets from the visual cortex to compare cell type detection. They estimated that the nuclear proportion of total cellular mRNA varies from $20 \%$ to over $50 \%$ for large and small pyramidal neurons, respectively. This contradicts the claim in the paper "90\% of RNA is cytosolic in the cortex."

\section{References}

1. Bakken TE, Hodge RD, Miller JA, Yao Z, et al.: Single-nucleus and single-cell transcriptomes compared in matched cortical cell types.PLoS One. 2018; 13 (12): e0209648 PubMed Abstract I Publisher Full Text

2. Patrick E, Taga M, Ergun A, Ng B, et al.: Deconvolving the contributions of cell-type heterogeneity on cortical gene expression.PLoS Comput Biol. 16 (8): e1008120 PubMed Abstract | Publisher Full Text

\section{Is the rationale for developing the new method (or application) clearly explained? Yes}

\section{Is the description of the method technically sound?}


Are sufficient details provided to allow replication of the method development and its use by others?

Yes

If any results are presented, are all the source data underlying the results available to ensure full reproducibility?

Yes

Are the conclusions about the method and its performance adequately supported by the findings presented in the article?

Partly

Competing Interests: No competing interests were disclosed.

We confirm that we have read this submission and believe that we have an appropriate level of expertise to confirm that it is of an acceptable scientific standard, however we have significant reservations, as outlined above.

\section{Comments on this article}

\section{Version 1}

Reader Comment 05 Aug 2021

Francisco Avila Cobos, Ghent University, Belgium

Never mind my previous comment, I found such file here:

https://github.com/ksosina/Strategies-for-cellular-deconvolution-in-human-brain-RNA-sequencingdata/blob/master/cell_type_data/Sample\%20estimates.xlsx

Thank you very much.

Francisco

Competing Interests: none

Reader Comment 05 Aug 2021

Francisco Avila Cobos, Ghent University, Belgium

Dear authors,

Would it be possible to provide an additional suppl. file with the cell type proportions (from DNAm, 
your gold standard) for the 223 samples? I was unable to find them among the sosina_extended .docx files.

Thank you.

Kind regards,

Francisco

Competing Interests: none

The benefits of publishing with F1000Research:

- Your article is published within days, with no editorial bias

- You can publish traditional articles, null/negative results, case reports, data notes and more

- The peer review process is transparent and collaborative

- Your article is indexed in PubMed after passing peer review

- Dedicated customer support at every stage

For pre-submission enquiries, contact research@f1000.com 